\title{
Photonic Jet Suitable for High Precision Contact Laser Surgery Applications in Water
}

\author{
Mohamed Karim Azizi \\ Department of Physics \\ Faculty of Sciences of Tunis \\ University of Tunis El Manar \\ Tunis, Tunisia \\ medkarim.azizi@gmail.com
}

\author{
Abdullah Alhumaidi Alotaibi \\ Department of Mathematics \\ Turabah University College \\ Taif University \\ Taif, Saudi Arabia \\ a.alhumaidi@tu.edu.sa
}

\begin{abstract}
The use of contact probes in surgical laser technologies (SLT) allows tissue contact without damage and enables tactile feedback during operations. Among the materials suitable for the manufacturing of chirurgical contact probes, sapphire has been widely used. Indeed, the optical properties of this material allow the formation of a high energy density localized region at the front of the contact probe, when used in air. However, in water, this focusing effect is very weak. In this work, the use of a cylindrical sapphire contact probe associated with a continuous (CW) Nd: Yag laser (at 1064nm) is proposed and studied, which provides, in water, a narrow and high-intensity beam (photonic jet). With the evolution of technology, this kind of surgery can be done remotely. Based on 5G technology, medical experts can bring their skills to remote other practitioners around the world. The obtained results show a linear dependence of the focal length and a linear dependence of the beam intensity of the photonic jet to the cylinder radius while the full width at half maximum of the photonic jet beam shows exponential decay dependence. Such a system could give rise to a new kind of optical scalpel to the ultraprecise laser surgery in water.
\end{abstract}

\section{Keywords-photonic jet; sapphire; scalpel; surgery}

\section{INTRODUCTION}

The concept of photonic jet [1] was presented for the first time in 2004. When a micro cylinder is illuminated by a plane wave, a beam (photonic jet) with a waist smaller than the classical diffraction limit is formed on the shadow-side surface of the cylinder. Another characteristic of the photonic jet is its low spatial distribution which leads to a high energy density localization and thus to a high intensity exceeding that of the incident wave. Until now [2-3], various studies and applications on these systems were carried out in microscopy [4] to improve image resolution, in spectroscopy [5-6] for the sensing of nanoparticles, or in biology [7]. Several works have treated the case of photonic nano-jets emerging from dielectric micro cylinders illuminated by plane waves. The intensity of the nano jet and its location depend on the diameter of the micro cylinder and the refraction of the index between the micro cylinder and the surrounding environment. To do this, one must first define the width and the spatial length of the nano jet. The interaction of an electromagnetic wave with a metallic or dielectric obstacle, allows having at well-defined dimensions a diffused light well localized in a space of rather small volume. This phenomenon is called a photonic jet $[8,9]$. This beam of high intensity, propagates over a distance of a few wavelengths with a section less than the wavelength. This small section is one of the essential characteristics of the photonic jet, since it is not possible to obtain it with a conventional optical device. This allows us to exploit the photonic jet to obtain optical instruments with a resolution lower than $\lambda / 2$. Photonic jet applications have been carried out for the detection of molecules [10], optical imaging, data storage [11], and high-resolution microscopy [12, 13]. The majority of these studies and applications were based on obtaining a photonic jet from the interaction of an electromagnetic wave with spherical $[14,15]$, cylindrical [16] or elliptical [17] obstacles [18].

Another area where the use of photonic jets is likely to make a significant contribution is the medical field and more precisely the field of surgery. In surgery, the need to make precise incisions without damaging or harming the tissue is the cause of the development of several types of scalpels [19-20]. Indeed, cut with a conventional scalpel requires mostly to force on tissues (pressure and pull). Also, for example, the use of a laser [21] can damage tissues at the edge of the cut (due to high temperature), which can result in longer and more painful healing. This is disadvantageous because we need a high-power density since the greater the power density, the more rapid the tissue response. Thanks to $5 \mathrm{G}$ technology, this kind of intervention can be done remotely, since $5 \mathrm{G}$ offers an even shorter reaction time (latency) and much better image quality than previous networks, thus limiting the risks of mistake in bringing more information to medical teams. $5 \mathrm{G}$ enables specialists to work together from different places due to its ability to offer high resolution images, enabling also specialists to monitor the console screen of the radiological device. By using the Terahertz band which currently is a band less used, we can go towards very high speeds and thanks to the integration of miniature and efficient antennas [22] in this frequency band.

In this paper, the use of a cylindrical sapphire contact probe associated to a continuous (CW) Nd: Yag laser (at 1064nm) which provides in water a narrow and high intensity photonic 
jet is theoretically demonstrated. Such a system could give rise to a new kind of optical scalpel to the ultra-precise laser surgery.

\section{THEORY}

The calculation model used is based on rigorous resolution of the Maxwell equations and allows determining the expressions of the field inside the cylinder as well as that of the diffracted field by the cylinder. Assuming that the fields are TE with a temporal variation of the form $\exp (-i \omega t)$, where $\omega$ is the angular frequency and $t$ the time, the incident, absorbed and diffracted fields are expressed in the form of Fourier-Bessel series. The expressions of the fields inside and outside the cylinder are then written in term of the Bessel functions as:

$$
E_{z, i}(r, \theta)=\sum_{m \in Z} c_{m} J_{m}\left(k_{i} r\right) e^{i m \theta}
$$

and

$$
E_{z, e}(r, \theta)=\sum_{m \in Z} a_{m} J_{m}\left(k_{e} r\right) e^{i m \theta}+\sum_{m \in Z} b_{m} H_{m}^{(1)}\left(k_{e} r\right) e^{i m \theta}
$$

where subscripts $i$ and $e$ indicate internal and external fields, $k_{i}$ and $k_{e}$ are wave vectors, $r$ and $\theta$ are the polar coordinates and $a_{m}, b_{m}$ and $c_{m}$ are coefficients that are determined after consideration of the conditions of continuity of the tangential components of the field at $r=R$ (radius of the cylinder). In the simulations the cylinder center is laid out in the $x-y$ plane (at the intersection of the two axes) and the light propagation is along the $x$ axis. The cylinder is made of sapphire crystal which is largely transparent to the Nd:Yag laser wavelengths. An incident plane wave with wavelength $\lambda=1064 \mathrm{~nm}$ is adopted. At this wavelength, the sapphire cylinder has a refractive index of $n_{c}=1.7545$ while the surrounding medium (water) has a refractive index $n_{w}=1.33$.

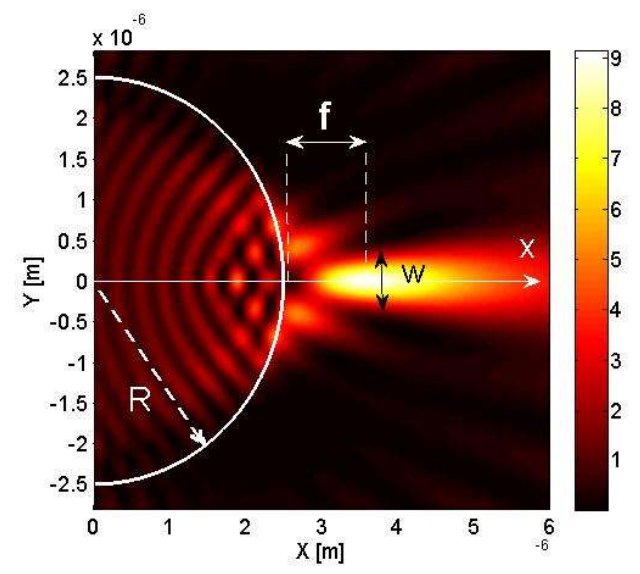

Fig. 1. Schematic representation of the focal and the waist of a photonic jet

As shown in Figure 1, the photonic jet can be described essentially by three parameters: its focal length $f$, its width at half maximum $w$ in the $x-y$ plane and its intensity Imax at the focal point. The focal length is considered as the distance from the surface of the cylinder to the point of maximum intensity along the $x$ axis. Considering a constant index contrast of 1.32, the cylinder radius is varied and we explore how this change affects the scattered field which forms the photonic jet.

\section{RESULTS AND DISCUSSION}

Figure 2 shows the electric field intensity map obtained for a cylinder of $R=2.5 \mu \mathrm{m}$ radius. From the shadow side surface of the illuminated cylinder, a photonic jet emerges with high intensity and a focal length $f=1.1 \mu \mathrm{m}$. This photonic jet is a result of the interference between the incoming plane wave and the scattered field from the cylinder. As depicted in Figure 3, the distribution of the intensity of the electromagnetic field along the propagation axis $x$ shows a maximum at $x=3.6 \mu \mathrm{m}$ which represents the focus point ( $\mathrm{F}$ in Figure 2). From this focus point, the intensity decreases continuously and reaches the value $\operatorname{Imax} / \mathrm{e}^{2}$ for a distance of $5.45 \lambda$ relative to the focal point $F$. This propagation of the electromagnetic beam over several wavelengths along the propagation axis with little divergence is a particularity of the photonic jets.

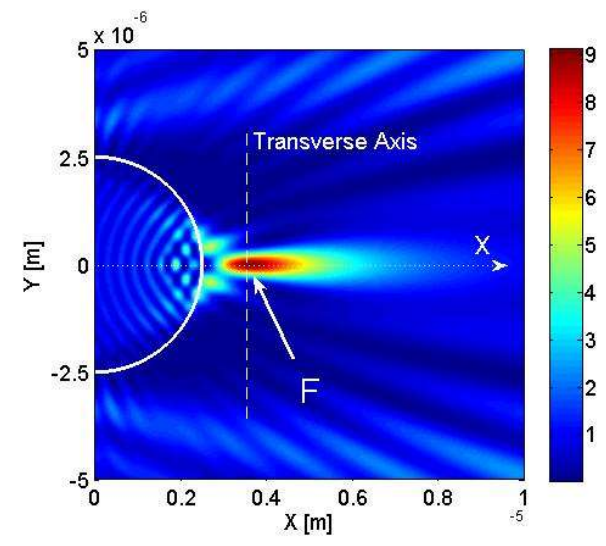

Fig. 2. Electric field intensity map $(R=2.5 \mu \mathrm{m})$. The photonic jet shows a maximum intensity at $F$ (focus). The Transverse Axis represents the axis that passes through $\mathrm{F}$ and is perpendicular to the $x$ direction.

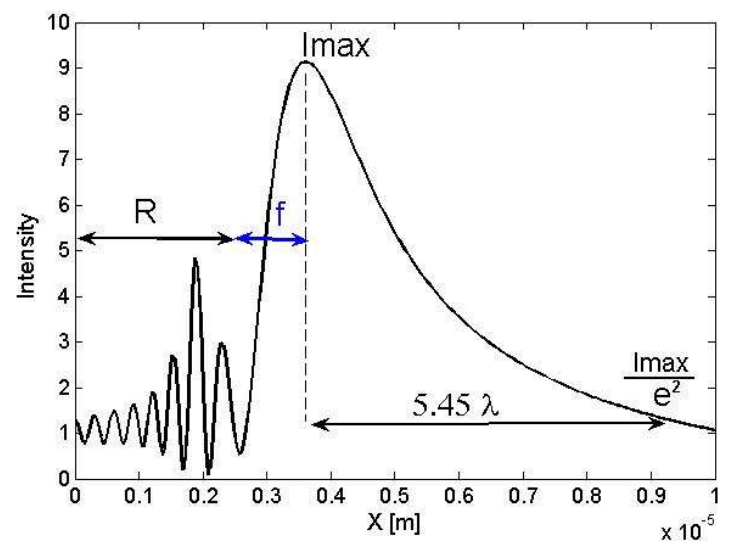

Fig. 3. Intensity distribution of the electromagnetic field along the propagation axis $x(R=2.5 \mu \mathrm{m})$. The photonic jet shows a longitudinal waist of $5.45 \lambda$

Figure 4 shows the distribution of the beam intensity along the transverse axis. As shown in this Figure, in the immediate vicinity of the focal point, this curve is symmetrical and welladjusted by a Lorentzian profile. The Full Width at Half Maximum (FWHM) of the photonic jet is then represented by the FWHM of this Lorentzian profile. The characteristic of this system is that the values of the FWHM are smaller than the 
wavelength. The photonic jet thus provides a good focusing with sub wavelength spot size. As depicted in Figure 5, the focal length, the intensity enhancement at the focus point and the FWHM of the photonic jet at the focus point depend on the cylinder radius. It is therefore necessary to find the value of the radius $R$, which optimizes these three parameters.

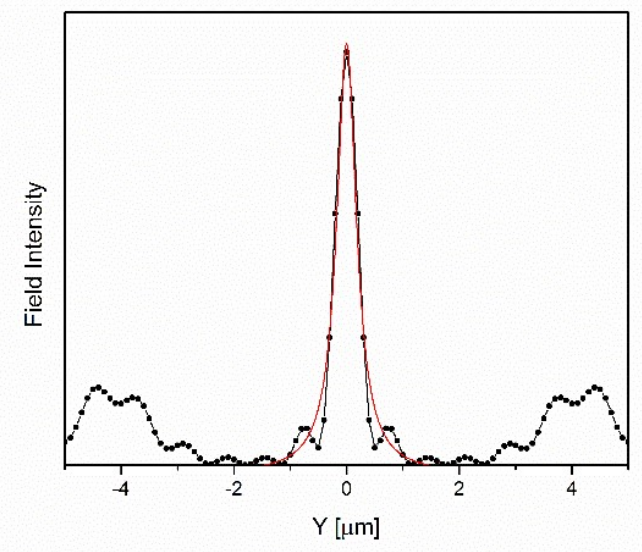

Fig. 4. Electromagnetic field intensity along the transverse axis (black dotted curve) with Lorentzian profile fitting (red curve)
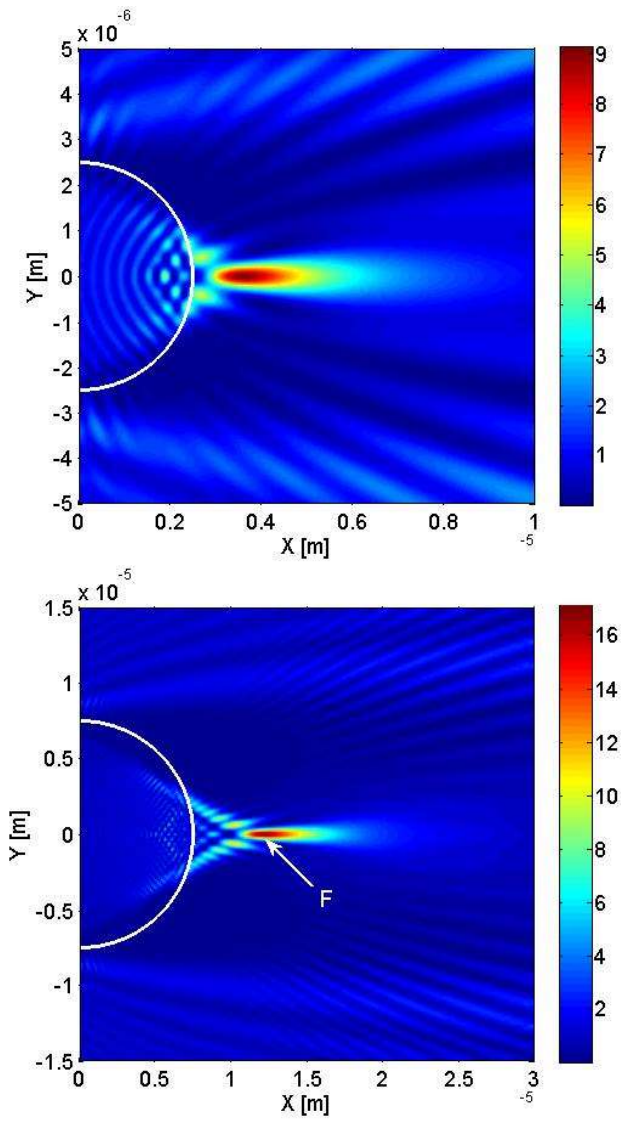

Fig. 5. Electric field intensity map $(R=2.5 \mu \mathrm{m}$ and $R=7.5 \mu \mathrm{m})$. The focal length, the intensity, and the FWHM at the focus point vary with $R$

\section{A. Focal Length Variation}

The variation of the focal length of the photonic jet was studied by varying the radius of the cylinder from $1 \mu \mathrm{m}$ to $10 \mu \mathrm{m}$. As shown in Figure 6, this variation is linear and has a slope equal to 0.73 . For larger values of the radius $R$, a problem of focus, which consists of the appearance of different points of equal intensity, appears (Figure 7).

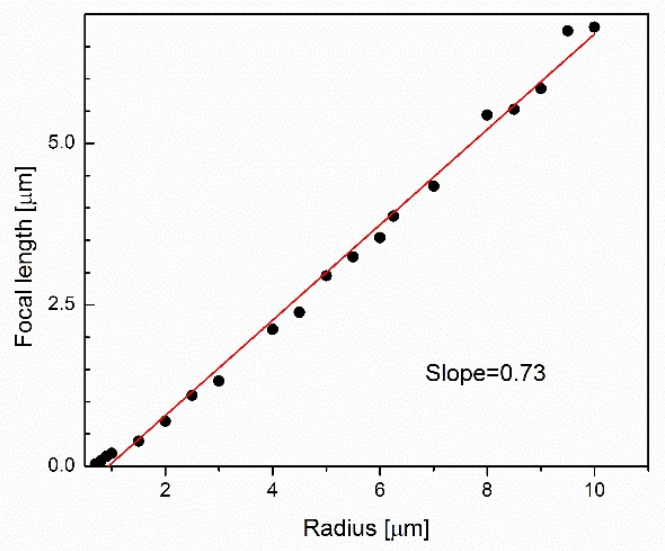

Fig. 6. Linear variation of the focal length versus cylinder radius

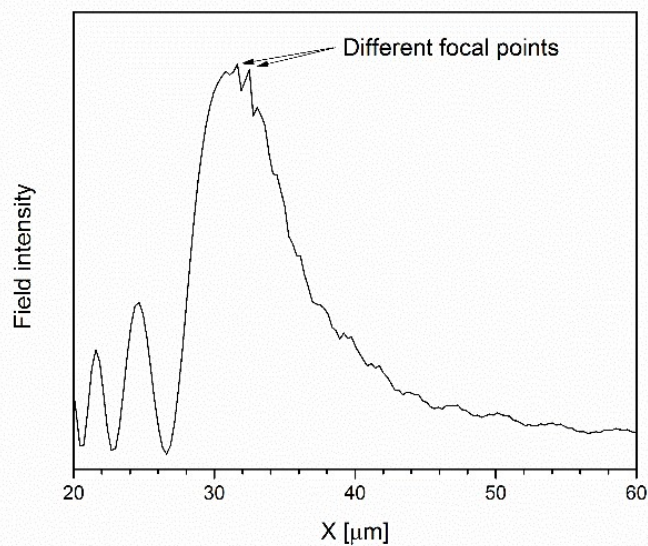

Fig. 7. Intensity distribution of the electromagnetic field along the propagation axis $x(R>10 \mu \mathrm{m})$. Focusing problem appears

Taking into account the fact that the wavelengths in the spectral 0.6 to $1.2 \mu \mathrm{m}$ are poorly absorbed and penetrate deeply into the tissues, a large focal length is thus not necessary in our case. This penetration is however limited by optical scattering and thus depends on the width at half height of the photonic jet.

\section{B. Intensity Variation}

The intensity is a key parameter in the interaction of an optical scalpel with tissues. Indeed, once the wavelength of the photonic jet and the tissues-optical coefficients are well chosen, evaporation of the tissue will depend on its thermal parameters as well as on the intensity of the photonic jet (energy density 
and duration of application). The simulations results show that as the focal length, the enhancement of the intensity of the photonic jet increases linearly with the radius (Figure 8).

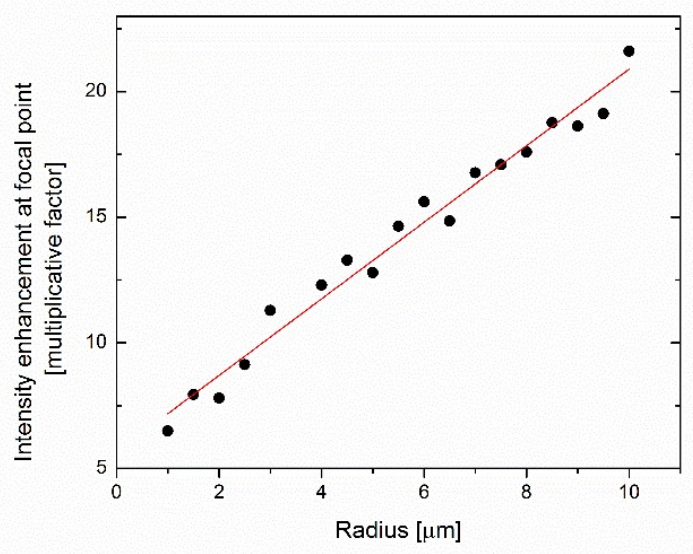

Fig. 8. Enhancement of the intensity at the focal point

\section{FWHM Variation}

As presented in Figure 9, the FWHM of the photonic jet decreases exponentially with cylinder radius to sub-wavelength values of the order of $\lambda / 10$. This allows maximum energy density over a very low area and then a rapid diffusion.

\section{Choice of Parameters}

The preceding results show that it would be appropriate to select a cylinder radius of between $6 \mu \mathrm{m}$ and $10 \mu \mathrm{m}$ because of the small change in FWHM in this interval. In this range, a sub wavelength spot size of about $\lambda / 10$ is provided which allows ultra-precise manipulation. In addition, this low value of the spot size minimizes thermal injury to adjacent tissues, for the considered intensities. Thus, choosing a radius value close to the lower bound of this interval seems the best choice.

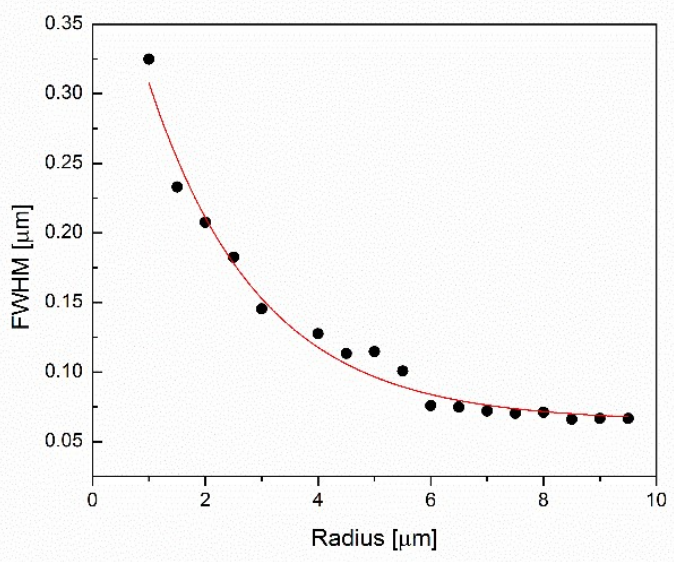

Fig. 9. Exponential decrease of the photonic jet FWHM vs cylinder radius

\section{CONCLUSION}

The use of a cylindrical sapphire contact probe associated to a continuous (CW) Nd:Yag laser (at 1064nm) was theoretically demonstrated which provides, in water, a narrow and high intensity photonic jet with spot size of about $\lambda / 10$. Such a system could give rise to a new kind of optical scalpel to the ultra-precise laser surgery. In the near future several applications will be able to be treated, such as detection of cancer in the health field, improvement of data storage in the digital field, engraving and welding in the industrial field.

\section{REFERENCES}

[1] Z. Chen, A. Taflove, V. Backman, "Photonic nanojet enhancement of backscattering of light by nanoparticles: A potential novel visible-light ultramicroscopy technique", Optics Express, Vol. 12, No. 7, pp. 1214 1220, 2004

[2] C Zaichun, Z Hengyu, H Minghui, "Ultra-long photonic jet by hemispherical micro-particles", in: Laser Science, Optical Society of America, 2015

[3] H. Mohseni, "Photonic jet and its applications in nano-photonics", in: Frontiers in Optics, Optical Society of America, 2015

[4] E. Betzig, J. K. Trautman, "Near-field optics: Microscopy, spectroscopy, and surface modification beyond the diffraction limit", Science, Vol. 257, No. 5067, pp. 189-195, 2005

[5] W. Guo, Z. B. Wang, L. Li, D. J. Whitehead, B. S. Luk'yanchuk, Z. Liu, "Near-field laser parallel nanofabrication of arbitrary-shaped patterns", Applied Physics Letters, Vol. 90, pp. 24-26, 20007

[6] X. Li, Z. Chen, A. Taflove, V. Backman, "Optical analysis of nanoparticles via enhanced backscattering facilitated by 3-D photonic nanojets", Optics Express, Vol. 13, No. 2, pp. 526-533, 2005

[7] H. Yang, M. Cornaglia, M. A. M. Gijs, "Photonic nanojet array for fast detection of single nanoparticles in a flow", Nano Letters, Vol. 15, No. 3, pp. 1730-1735, 2015

[8] M. K. Azizi, H. Baudrand, T. Elbellili, A. Gharsallah, “Almost periodic lumped elements structure modeling using iterative method: Application to photonic jets and planar lenses", Progress In Electromagnetics Research M, Vol. 55, pp. 121-132, 2017

[9] M. S. Kim, T. Scharf, S. Muhlig, C. Rockstuhl, H. P. Herzig, "Engineering photonic nanojets", Optics Express, Vol. 19, No. 11, pp. 10206-10220, 2011

[10] Z. Wang W. Guo, L. Li, B. Luk'yanchuk, A. Khan, Z. Liu, Z. Chen, M. Hong, "Optical virtual imaging at $50 \mathrm{~nm}$ lateral resolution with a whitelight nanoscope", Nature Communications, Vol. 2, No. 1, pp. 1-6, 2011

[11] V. R. Dantham, P. B. Bisht, C. K. R. Namboodiri, "Enhancement of Raman scattering by two orders of magnitude using photonic nanojet of a microsphere", Journal of Applied Physics, Vol. 109, No. 10, Article ID 103103, 2011

[12] S. C. Kong, A. Sahakian, A. Taflove, V. Backman, "Photonic nanojetenabled optical data storage", Optics Express, Vol. 16, No. 18, pp. 13713-13719, 2008

[13] A. Heifetz, S. C. Kong, A. V. Sahakian, A. Taflove, V. Backman, "Photonic nanojets", Journal of Computational and Theoritical Nanoscience, Vol. 6, No. 9, pp. 1979-1992, 2009

[14] Z. Chen, A. Taflove, V. Backman, "Photonic nanojet enhancement of backscattering of light by nanoparticles: A potential novel visible-light ultramicroscopy technique”, Optics Express, No. 12, No. 7, pp. 12141220,2004

[15] X. Li, Z. Chen, A. Taflove, V. Backman, "Optical analysis of nanoparticles via enhanced backscattering facilitated by 3-D photonic nanojets", Optics Express, Vol. 13, No. 2, pp. 526-533, 2005

[16] S. Lecler, Y. Takakura, P. Meyrueis, "Properties of a 3D photonic jet", Optics Letters, Vol. 30, No. 19, pp. 2641-2643, 2005

[17] A. V. Itagi, W. A. Challener, "Optics of photonic nanojets", Journal of the Optical Society of America A, Vol. 22, No. 12, pp. 2847-2858, 2005 
[18] N. Ammar, T. Aguili, H. Baudrand, B. Sauviac, B. Ounnas, "Wave concept iterative process method for electromagnetic or photonic jets: Numerical and experimental results", IEEE Transactions on Antennas and Propagation, Vol. 63, No. 11, pp. 4857-4867, 2015

[19] T. Lu, W. Zhang, F. Chen, Z. Liu, "Microliquid jet induced by tunable holmium laser: A potential microsurgery scalpel", Microfluidics and Nanofluidics, Vol. 20, No. 1, Article ID 10, 2016

[20] M. C. Chiang, C. C. Huang, "Optically-guided scalpel with lightscattering module for carpal tunnel surgical procedure via minimally invasive surgery", Bio-medical Materials and Engineering, Vol. 26, No. S1, pp. S173-S179, 2015

[21] S. M. A. Ghaly, M. O. Khan, "Design, simulation, modeling, and implementation of a square helmholtz coil in contrast with a circular coil for MRI applications", Engineering, Technology \& Applied Science Research, Vol 9, No. 6, pp. 4990-4995, 2019

[22] M. Amine Ksiksi, M. Karim Azizi, H. Ajlani, A. Gharsallah, 'Frequency reconfigurable square patch antenna based on graphene for telecommunication systems", Engineering Technology \& Applied Science Research, Vol. 9, No. 5, pp. 4846-4850, 2019 